\title{
Energy Spectrum of InAs Quantum Dots in GaAs/AlAs Superlattices
}

\author{
R. Nedzinskas ${ }^{a, *}$, B. ČechaviČIUs ${ }^{a}$, J. KAVAliauskas ${ }^{a}$,

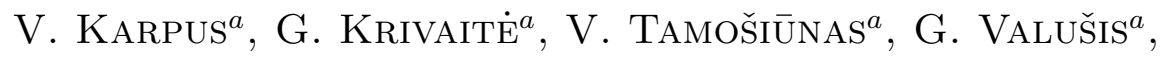 \\ F. Schrey ${ }^{b}$, K. UnterRAineR ${ }^{b}$ AND G. Strasser ${ }^{c}$ \\ ${ }^{a}$ Semiconductor Physics Institute
}

A. Goštauto 11, LT-01108, Vilnius, Lithuania

${ }^{b}$ Institut für Photonik, Technische Universität-Wien

A-1040, Vienna, Austria

${ }^{c}$ Institut für Festkörperelektronik, Technische Universität-Wien

Floragasse 7, A-1040 Vienna, Austria

\begin{abstract}
Photo- and contactless electroreflectance spectroscopies were applied to study optical properties and electronic structure of GaAs/AlAs superlattice systems with embedded InAs quantum dots. The observed interband transitions related to the quantum dot ground and excited states, as well as optical transitions in the combined system formed by the InAs wetting layer and GaAs/AlAs superlattice are discussed.
\end{abstract}

PACS numbers: 78.67.Hc

\section{Introduction}

In recent years, GaAs/AlAs superlattice (SL) structures with embedded InAs quantum dots (QDs) have attracted considerable attention due to applications for infrared photodetectors. The interest in $\mathrm{QD} / \mathrm{SL}$ combined systems is based on the possibility to tune optical transition energies by varying SL period as well as by changing QD growth conditions [1]. The suitability of combined QD/SL systems for the use as mid-infrared photodetectors with reduced dark current was confirmed in [2]. Moreover, it was shown that QDs together with their wetting layer (WL) modify noticeably the energy structure of GaAs/AlAs SL. To find optimal design for potential applications, knowledge about optical properties and electronic structure of combined QD/SL systems is essential.

*corresponding author; e-mail: ramunas@pfi.lt 
In this work, we report the first experimental study of interband transitions and electronic levels, using photoreflectance $(\mathrm{PR})$ and contactless electroreflectance (CER) spectroscopy methods, in InAs/GaAs/AlAs QD-SL system. Also, photoluminescence (PL) spectra were measured for comparison. The interpretation of experimental data was based on calculations of InAs QD energy states using nextnano ${ }^{3}$ simulator [3].

\section{Samples and experimental techniques}

Quantum dot structures studied in Refs. [1, 2] consist of periodically arranged InAs QD layers, embedded in GaAs matrix, with period of $10 \mathrm{~nm}$ (sample 469 ) or in a GaAs/AlAs SL with period of $14 \mathrm{~nm}$ (sample 577). In the latter case, dot layers are placed at $1 \mathrm{~nm}$ distance from $1 \mathrm{~nm}$ thickness AlAs barriers. Both sample schemes are shown in Fig. 1. As-grown QD active layers were sandwiched between $n^{+}$-doped $\left(n \approx 10^{18} \mathrm{~cm}^{-3}\right)$ GaAs contact layers. For optical measurement the top $n^{+}$-GaAs layer was etched in order to merge surface depletion layer to the QDs ensemble and to increase the electromodulation efficiency.
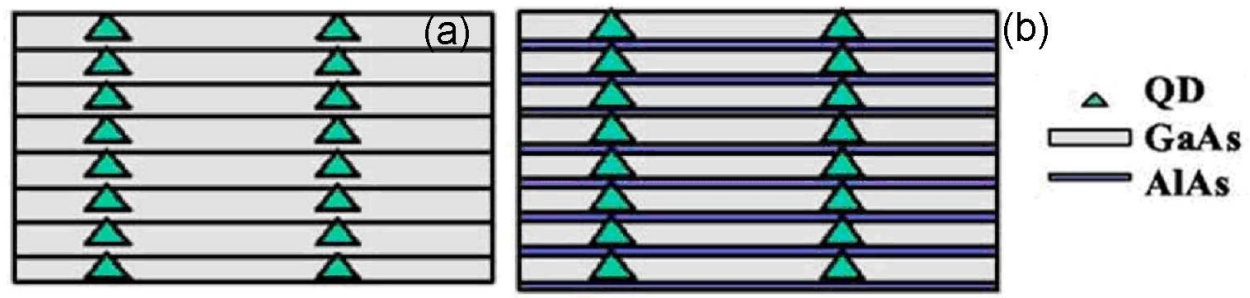

Fig. 1. Schematic representation of multiple QD structures studied: sample 469 (a) and sample 577 (b).

For the PR studies, a He-Ne $(632.8 \mathrm{~nm})$ or LED $(470 \mathrm{~nm})$ light beams as the excitation sources were employed [4]. In the CER experiment, a condenserlike system was utilized. An ac modulation voltage in the range of 300-500 V was applied to top transparent electrode to provide modulating field. All the experiments were performed at room temperature.

\section{Experimental results and discussion}

Room temperature PR spectrum for the sample 577 is shown in Fig. 2a. Arrows indicate the transition energies obtained from fitting the PR data with the first derivatives of the Gaussian line shape [4]. The PR spectrum exhibits the GaAs band gap bulk-like feature at $1.424 \mathrm{eV}$ from the undoped region of a buffer layer. In the spectral region $0.9-1.3 \mathrm{eV}$ the broadened features related to interband transitions in the QDs are seen. A low-energy PR feature is located almost exactly at the maximum of the broad PL line and corresponds to the ground-state transitions. The dominant broadening comes from fluctuations in 
the dots sizes of the stacked structure. As follows from the experimental data and energy state simulation, there are likely three bound states in the conduction and valence band of InAs QDs, respectively.
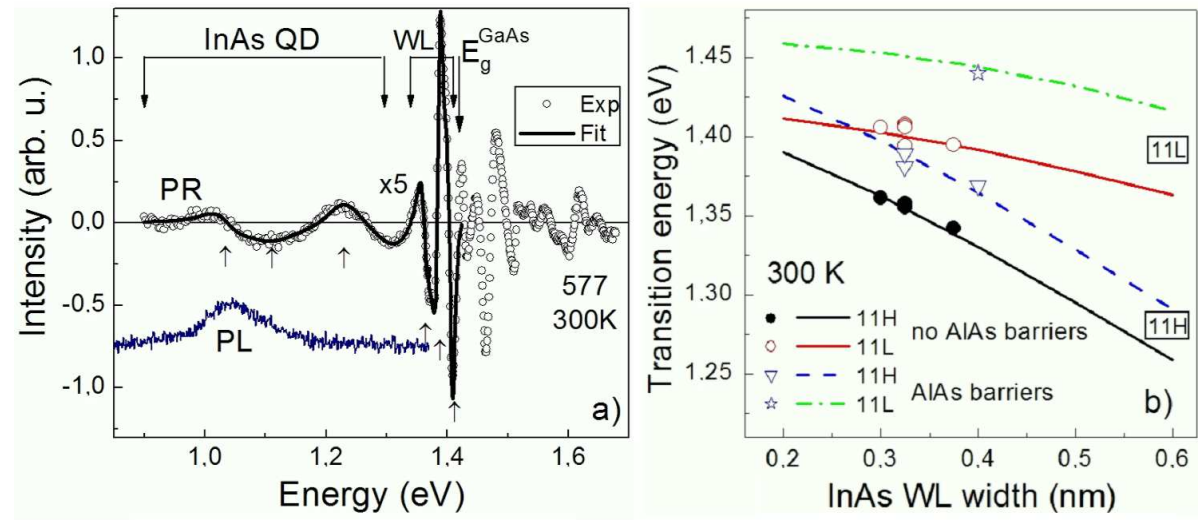

Fig. 2. (a) Comparison of PR and PL spectra of multilayer QD structure for sample 577. The arrows indicate optical transition energies obtained from the fitting. (b) WLQW interband transition energies versus InAs WL thickness for QD structures with and without AlAs barriers: symbols — experiment, lines — calculations.

Further, sharp resonances in the $1.36-1.41 \mathrm{eV}$ region correspond to the lowest energy excitonic transitions in the combined InAs WL and GaAs/AlAs SL system. Splitting is the characteristic feature of these heavy-(11H) and light-hole (11L) related optical transitions. The transition energies indicate that it cannot be explained exclusively in terms of electronic coupling between WLs [5]. In order to account for the measured PR features, it was assumed tentatively that WL thickness varies across the QD stacks due to strain coupling [5]. It should be noted that such splitting of WL related interband transitions follows also from the PR/CER spectra of QD stacks formed in GaAs matrix for the sample 469 (this data is not shown here).

The nature of particular transitions was identified by electronic state simulation. To get exact magnitudes of WL thickness, we have compared the measured $11 \mathrm{H}$ and $11 \mathrm{~L}$ transition energies with those calculated in the effective mass approximation of the envelope function approach. The dependences of wetting layerquantum well (WL-QW) interband transition energies versus InAs WL thickness for QD structures with (sample 577) and without (sample 469) AlAs barriers are presented in Fig. 2b. The comparison of these results indicates clearly that InAs QDs and WL modify the energy structure of GaAs/AlAs SL. In particular, the ground states of the SL subbands are shifted markedly to lower energy and coincide with the InAs WL energy subband. Vice versa, embedding of InAs QDs into GaAs/AlAs SL shifts the WL related states to higher energies. As was mentioned 
above, the splitting of WL states demonstrates the strain modulation in the stacked QD layers. As follows from experimental results and effective mass square-like QW energy level calculations (Fig. 2b), the InAs WL thickness decreases slightly across QD stacks from about 1.3 to 1 monolayer (ML).

In conclusion, CER and PR spectroscopies have been used to study optical transitions in $\mathrm{QD} / \mathrm{SL}$ system. The interband transitions related to the ground state, excited states in InAs QDs, and minibands of InAs/GaAs/AlAs SL were observed. The results indicate that InAs QDs and WL modify the energy structure of GaAs/AlAs SL.

\section{Acknowledgments}

We kindly thank Stefan Birner for helping us with the nextnano ${ }^{3}$ software. The work was supported, in part, by the Lithuanian State Science and Studies Foundation under contract C-07004 and the EU POISE project.

\section{References}

[1] L. Rebohle, F.F. Schrey, S. Hofer, G. Strasser, K. Unterrainer, Appl. Phys. Lett. 81, 2079 (2002).

[2] F.F. Schrey, L. Rebohle, T. Mueller, G. Strasser, K. Unterrainer D. Nguyen, N. Regnault, R. Ferreira, G. Bastard, Phys. Rev. B 72, 15310 (2005).

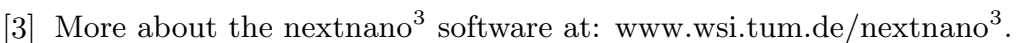

[4] B. Čechavičius, J. Kavaliauskas, G. Krivaitè, D. Seliuta, G. Valušis, M. Halsall, M. Steer, P. Harrison, J. Appl. Phys. 98, 023508 (2005).

[5] A. Winzer, R. Goldhahn, G. Gobsch, H. Heidemeyer, O. Schmidt, K. Eberl, Physica E 13, 289 (2002). 